\title{
Comparative evaluation of the sleep quality in male verses female students of Nursing at school of nursing and midwifery of MBU
}

\author{
Morteza Alibakhshi-kenari \\ Medical Sciences and Health Services Department of Martyr Beheshti University (MBU) of Tehran, Iran
}

Email address:

Morteza.Alibakhshikenari@gmail.com

To cite this article:

Morteza Alibakhshi-kenari. Comparative Evaluation of the Sleep Quality in Male Verses Female Students of Nursing at School of Nursing and Midwifery of MBU. American Journal of Nursing Science. Vol. 3, No. 3, 2014, pp. 26-33. doi: 10.11648/j.ajns.20140303.11

\begin{abstract}
Aims: Traditionally science students exhibit poor sleep quality and impaired academic and professional performances due to high sleep deprivation and high incidences of daytime dysfunctions. The present study was aimed to evaluate the sleep quality in nursing students from the School of nursing and midwifery, Shahid Beheshti University of Medical Sciences (MBU) in Tehran. Study design: Sleep quality was evaluated by filling out the Pittsburgh Sleep Quality Index (PSQI). Place and Duration of Study: School of nursing and midwifery, Shahid Beheshti University of Medical Sciences (MBU) in Tehran, during 1 November, 2012 to 1 May, 2014. Methodology: 133 nursing students were divided into different groups (on the basis of age; group to 4); each group was further divided into two groups based on sex. Mean values of the score obtained for each PSQI category were analyzed by the Student t-test and 95\% of Confidence of Intervals, to investigate the statistical differences among the male verses female groups and the correlation between the group and its respective score. Results: Findings showed that nursing students presented a fairly poor subjective sleep quality. Mean scores of sleep quality (PSQI category 1), sleep latency (PSQI category 2), sleep duration (PSQI category 3) and sleep efficiency (PSQI category 4) of female students were found significantly high in comparison to male students, while mean score of sleep disturbances (PSQI category 5), use of hypnotics/anti33 depressants (PSQI category 6) and daytime dysfunction (PSQI category 7) were non-significantly different among female and male students. Conclusion: The poor sleep quality in nursing students suggests that authorities should have specific guidance on sleep hygiene and should provide support for students presenting disturbances in day-time activities.
\end{abstract}

Keywords: Disorders of Excessive Somnolence, Education, Nursing Students Sleep, Sleep Disorders, Pittsburgh Sleep Quality Index (PSQI)

\section{Introduction}

National Institutes of Health (NIH) have been identified, adolescents 44 and young adults (ages 12 to 25 years) as a population at high risk for problem sleepiness based on "evidence that the prevalence of problem sleepiness is high and increasing with particularly serious consequences," [1]. Sleep is defined on the basis of behavioral and physiological criteria dividing it into two states: non rapid eye movement (NREM) sleep which is subdivided into three stages (N1, N2, N3); and rapid eye movement (REM) sleep characterized by rapid eye movements, muscle atonia and desynchronized EEG (electroencephalograph). Circadian rhythm of sleep-wakefulness is controlled by the master clock located in the suprachiasmatic nuclei of the hypothalamus. The neuroanatomical substrates of the NREM sleep are located principally in the ventrolateral preoptic nucleus of the hypothalamus and those of REM sleep are located in pons. A variety of significant physiological changes occur in all body system and organs during sleep as a result of functional alterations in the autonomic and somatic nervous systems [2]. Sleep is an active process which is functionally related to the wakefulness and the rhythmic alternation between these two states [3]. The metabolic cycle consisting of sleep, wakefulness, digestion, hormone production, thermal regulation and other daily processes or circadian rhythm or biological clock is regulated by processes in the brain. An optimal wakefulness state is reached when an adult sleeps 7 to $8 \mathrm{~h}$ within a period of $24 \mathrm{~h}$, with nocturnal awakenings 
up to $5 \%$ of the total sleep time. Therefore, the adult sleep pattern should include $30 \%$ of dreams, $20 \%$ of deep sleep and $50 \%$ of light sleep [4-5] In the absence of environmental perception, the joint action of several nervous structures regulates this sleep state, which is also affected by environmental and endogenous factors. Among them, the social factors seem to be most important, such as work schedules and recreation. Besides this environmental synchronization, the sleep-wakefulness cycle is endogenously generated and regulated by the supraquiasmatic nucleus in the hypothalamus, which is considered the biological clock in mammalian [5]. Sleep quality can be estimated by some quantitative or subjective components from the Pittsburgh Sleep Quality Index (PSQI), such as sleep duration, sleep latency, sleep disturbances and daytime dysfunctions [6]. PSQI is commonly applied to evaluate the sleep quality in various studies [7]. The reduced sleep duration in students triggers an irregular pattern of the sleep-wakefulness cycle, which is characterized by large sleep latency on weekends and by short sleep duration on weekdays. This irregular cycle usually increases the incidence of sleep disturbances and daytime dysfunctions, such as excessive daytime sleepiness [7]. Both sleep disturbances and daytime dysfunctions evoke negative impacts on the activities carried out during the day by the students and contribute to impair their academic performance [8]. Sleep disturbances have significant implications for the individual, the family and the society. The quality of sleep directly affects the quality of waking life, including mental sharpness, productivity, emotional balance, creativity, physical vitality, and even body weight. No other activity delivers so many benefits with so little effort. Poor life quality may induce autonomic dysfunction, poor academic or professional performance of the students and may increase the incidence of psychiatric disorders and decrease the wakefulness, with further damages in the personal security and subsequent increase in accidents. Moreover, sleep disturbances may become risk factors for clinical and psychiatric disorders [9].

In students, some sleep disturbances, such as insomnia and arousal, have been associated with clinical depression and minor psychiatric disorders [10], attention deficit and poor learning development [11]. Excessive daytime sleepiness has been described as the main daytime dysfunction in the students. Daytime sleepiness was prevalent among $18.9 \%$ of the medical students from the University of Brasilia (Brazil) [12], 22.4\% of the students from the King Saud University (Saudi Arabia) [13], 51.5\% of the students from the Federal University of Goiás (Brazil) $[7,14]$, and $35.5 \%$ of the students from the International Medical University (Malaysia) [15]. Moreover, excessive daytime sleepiness in students has been correlated with short sleep duration [13], poor academic performance [12], minor psychiatric disorders [10], sleep disorders [16], depressive symptoms [17] and poor sleep quality [7]. Considering the poor sleep quality in students [7-12], the harmful effects on the student mental functions [17] and learning ability [11], can deteriorate the academic performance [18]. There is paucity of data on sleep quality of students in Pakistan presently. Therefore the present study was designed to evaluate the sleep quality in nursing students from Shahid Beheshti University of Medical Sciences and compare it with female verses male students. Further the evaluation which 97 could provide the guideline for the implementation of the programs to aid the students, in order to promote sleep hygiene.

\section{Materials and Methods}

A prior approval was obtained from Research Ethics Committee of MBU in Tehran to conduct the present study.

\subsection{Subjects}

A total number $(n)$ of 133 students of both male and female, aged 16-20 years, were included in this study. Students were enrolled in the semester 4 in the Shahid Beheshti University, Tehran, Iran. Participants were divided into two different groups i.e. male $(\mathrm{nm}=56)$ and female $(\mathrm{nf}$ $=77$ ). Both male $(\mathrm{M})$ and female $(\mathrm{F})$ groups were further divided into four groups each according to their ages and the random sampling. Group M1 $(\mathrm{nm} 1=13)$ and $\mathrm{F} 1(\mathrm{nfl}=20)$ (age: $16-17$ years), M2 $(\mathrm{nm} 2=14)$ and F2 $(\mathrm{nf} 2=20)$ (age: $>17-18$ years), M3 $(\mathrm{nm} 3=14)$ and F3 $(\mathrm{nf} 3=19)$ (age: $>18-19$ years), M4 $(\mathrm{nm} 4=15)$ and F4 $(\mathrm{nf} 4=18)$ (age: $>19-20$ years). All the students included in the study were signed an Informed Consent [5].

\subsection{Evaluation of Sleep Quality}

Sleep quality was evaluated by filling out a questionnaire PSQI (Appendix A), comprising of nineteen (19) self-rated questions; grouped into seven categories i.e. (1) sleep quality, (2) sleep latency, (3) sleep duration, (4) habitual sleep efficiency, (5) sleep disturbances, (6) use of hypnotics and/or anti119 depressants, and (7) daytime dysfunction. Each category score ranged from 0-3 points; score 0 (aggregated score 0) meaning "very good", the score 1 (aggregated score $0 \geq 7$ ) meaning "fairly good", the score 2 (aggregated score $7 \geq 14$ ) meaning "fairly bad" and the score 3 (aggregated score $14 \geq 21$ ) meaning "very bad". Sum of the scores from the seven categories yielded an aggregated score that ranges from $0-21$ points. Higher the aggregated score, the worse the sleep quality [5]. The PSQI has been widely used to evaluate the sleep quality, since it is internationally reliable and appropriately standardized for retrospective use [19].

\subsection{Statistical Analysis}

Data were presented as the mean \pm standard error of mean (S.E.M.) of the aggregated score obtained for each PSQI category in both male and female groups of the students. SPSS 19 (Statistical Package for the Social Sciences) was used to analyze the data. This analysis was carried out by the application of Student t-test, that was 
considered as statistical significance $P<0.05$. A $95 \%$ confidence interval of proportions was determined as follows:

$$
\mathrm{p} \pm(\sqrt{ } \mathrm{pq} / \mathrm{n}) \times \mathrm{Zd} / 2(20)
$$

where:

$\mathrm{p}=$ Mean aggregated score different groups from the PSQI

$\mathrm{q}=1-\mathrm{p}$

$\mathrm{Z}=$ Standard normal variate $(1.96)$

$\mathrm{d}=$ Degree of freedom [20]

\section{Results}

Total score of all seven categories from PSQI of both male and female nursing students was found $14.6125 \pm$ 1.38 out of 21 (Score point $=2.09$ ). A significant difference $(P<0.005)$ was observed by comparing total mean score (sum of all categories of PSQI) of all female (16.3675 \pm $1.15, \mathrm{nf}=77$ ) participants against total mean score (sum of all categories of PSQI) of all male $(12.8575 \pm 1.02, \mathrm{~nm}=56)$ participants $(\mathrm{P}=0.0305$, CI $95 \%=-6.685494$ to -0.334506 , Figure 1). Sum of mean score of all seven categories of PSQI (aggregated score) of different female groups were compared with similar age male groups. A significant difference $(P<0.005)$ was observed by the comparison of aggregated score of $\mathrm{F} 1$ verses $\mathrm{M} 1 \quad(\mathrm{p}=0.0337,95 \% \mathrm{CI}=-$ 6.9227 to -0.2973$), F 2$ verses $\mathrm{M} 2$ ( $\mathrm{p}=0.0237, \mathrm{CI}=-7.8387$ to $-0.6013)$ group. Comparison of aggregated score of F3 with M3 ( $\mathrm{p}=0.1071,95 \% 151 \mathrm{CI}=-7.3778$ to 0.7578$)$ and $\mathrm{F} 4$ with M4 ( $\mathrm{p}=0.1302,95 \% \mathrm{CI}=-8.1147$ to 1.0947$)$ did not show any significant difference (Figure 2). Figure 3 presents the score point of different categories of PSQI of nursing students.

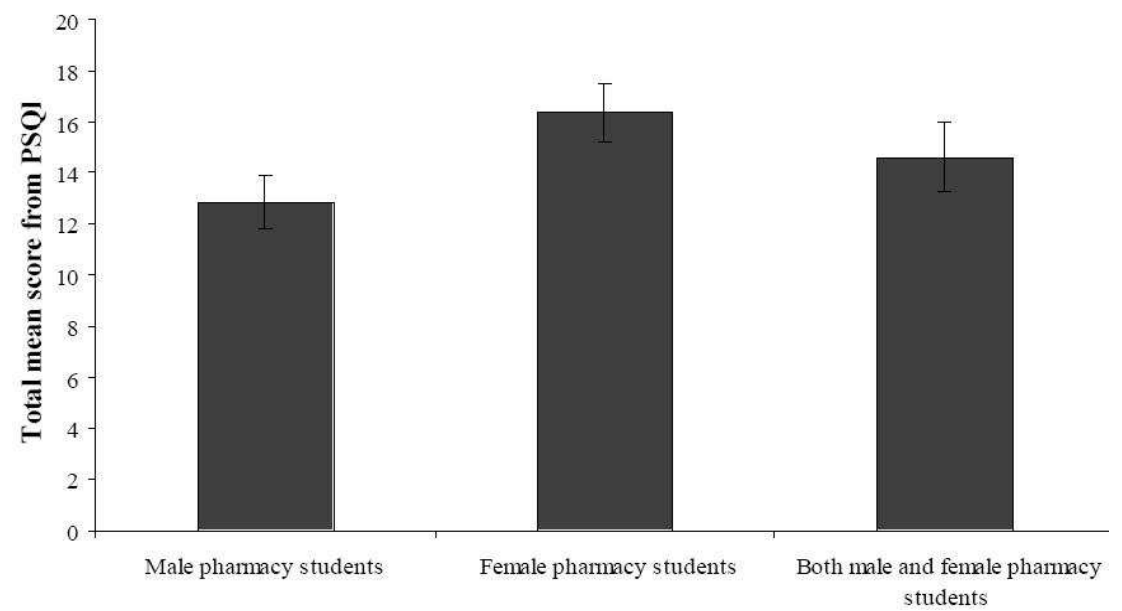

Fig. 1. Comparison of sum of score from all categories of PSQI of all male verses female nursing students.

PSQI: Pittsburgh Sleep Quality Index. Male nursing students presented a fairly bad sleep quality by achieving score point 2 (total mean score $1627 \geq 14$ ) while female nursing students showed a very bad sleep quality by achieving score point 3 (total mean score $14 \geq 21$ ). Therefore female nursing students showed a poorer level of sleep quality versus male nursing students. Overall score point of both male and female is near to 2 indicated a fairly bad level of sleep quality of nursing students.

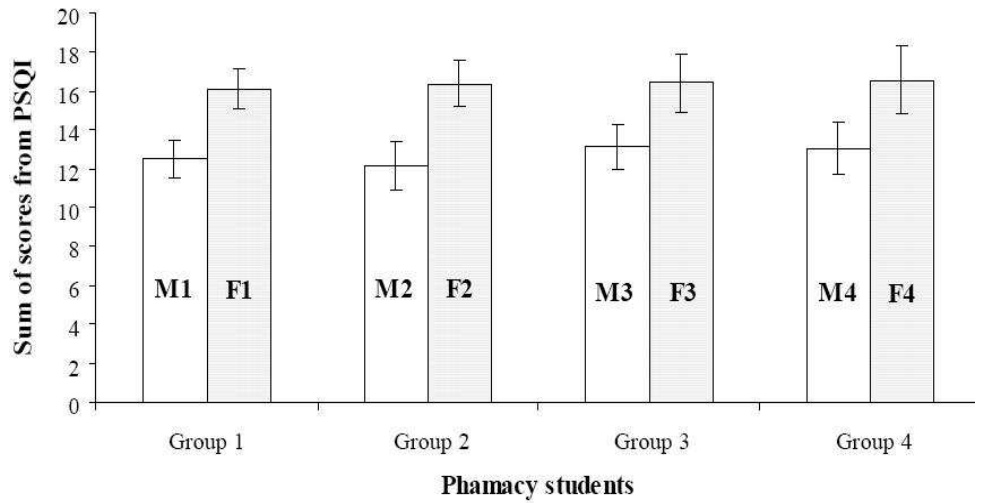

Fig. 2. Comparison of sum of mean score from all categories of PSQI of different groups of male verses female nursing students.

PSQI: Pittsburgh Sleep Quality Index, Group 1: M1 (male) and F1 (female): age, 16-17 years; Group 2: M2 (male) and F2 (female): age, >17-18 years; Group 3: M3 (male) and F3 (female): age, $>18-19$ years; Group 4: M4 (male) and F4 (female): age, $>19-20$ years. Different age male nursing students groups (M1, M2, M3 and M4) presented a fairly bad sleep quality by achieving score point 2 (total mean score $7 \geq 14$ ). Different age female nursing students groups (F1, F2, F3 and F4) showed a very bad level of sleep quality by achieving score point 3 (total mean score 14 $\geq 21$ ). Female nursing students showed a poorer level of sleep quality versus male nursing students. 


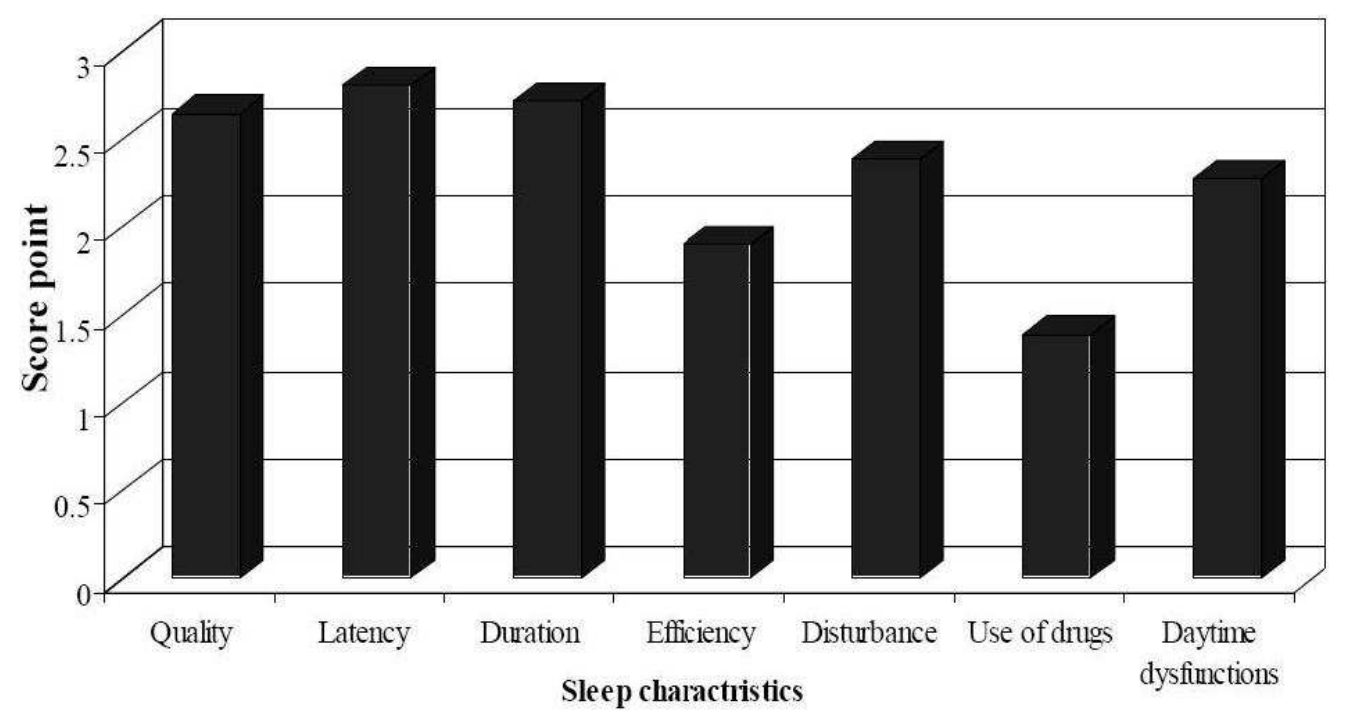

Fig. 3. Score point of all categories of PSQI of nursing students.

PSQI: Pittsburgh Sleep Quality Index, Use of drugs: use of hypnotics and/or anti-depressants. nursing students presented very bad sleep quality, sleep latency, sleep duration, sleep disturbances and daytime dysfunctions (score point more than 2) while sleep efficiency and use of hypnotics and/or antidepressants were judged fairly bad (score point near to 2 ) among students.

\subsection{Sleep Quality (PSQI Category 1)}

Mean score of sleep quality (PSQI category 1) of different female groups (F1-F4) were found significantly different $(P<0.005)$ when compared with similar age male groups (M1-M4) respectively (Table 1$)$. However male groups (M1-M4) of nursing students were insignificantly different from each other or from the values of M1 group. Mean score values from this PSQI category 1, obtained from F1, F2, F3, and F4, groups did not differ from each other or compared with the values of group F1 (Table 1).

Table 1. Comparison of sleep quality, aggregated score from the PSQI category 1 among male verses female nursing students

\begin{tabular}{lllll}
\hline Groups & Sex & Aggregated score & p-value & CI 95\% \\
\hline M1 (13) & Male & $2.02 \pm 0.267$ & & \\
F1 (20) & Female & $3.12 \pm 0.341^{*}$ & 0.0276 & -2.07053 to \\
M2 (14) & Male & $2.07 \pm 0.238$ & & -0.12947 \\
F2 (20) & Female & $3.10 \pm 0.322^{*}$ & 0.0239 & -1.91467 to \\
M3 (14) & Male & $2.09 \pm 0.224$ & & -0.14533 \\
F3 (19) & Female & $3.02 \pm 0.323^{*}$ & 0.0357 & -1.79370 to \\
M4 (15) & Male & $2.01 \pm 0.225$ & & -0.06630 \\
F4 (18) & Female & $3.14 \pm 0.356^{*}$ & 0.0156 & -2.03020 to \\
\hline
\end{tabular}

Pittsburgh Sleep Quality Index, Values are mean \pm M.S.E.

M2 (age $>17-18$ years), M3 (age $>18-19$ years) and M4 (age $>19-20$ years) groups were compared with M1 (age 16-17 years) group. F2 (age $>17-18$ years), F3 (age $>18-19$ years), and F4 (age $>19-20$ years), groups were compared with F1 (age 16-17 years), group. Female (F) groups were compared with respective (similar age) male (M) groups.

\subsection{Sleep Latency (PSQI Category 2)}

Table 2 summarized the mean score values of sleep latency of participant of this study. The comparison of female groups to respective similar age male groups showed significantly different $(P<0.005)$ score (Table 2$)$. Score values obtained from M2, M3 and M4 found insignificantly different in comparison to the values of M1. Similarly sleep latency score values of female F2, F3 and F4 groups remained insignificantly different from F1 group.

Table 2. Comparison of sleep latency, aggregated score from the PSQI category 2 among male verses female nursing students

\begin{tabular}{lllll}
\hline Groups & Sex & Aggregated score & p-value & CI 95\% \\
\hline M1 (13) & Male & $2.02 \pm 0.270$ & & \\
F1 (20) & Female & $2.82 \pm 0.232 *$ & 0.0341 & -1.53621 to \\
M2 (14) & Male & $2.07 \pm 0.144$ & & -0.06379 \\
F2 (20) & Female & $2.84 \pm 0.185^{*}$ & 0.0046 & -1.28399 to \\
M3 (14) & Male & $2.08 \pm 0.174$ & & -0.25601 \\
F3 (19) & Female & $2.85 \pm 0.161^{*}$ & 0.0031 & -1.25918 to \\
M4 (15) & Male & $2.12 \pm 0.175$ & & -0.28082 \\
F4 (18) & Female & $2.86 \pm 0.279^{*}$ & 0.0401 & -1.44435 to \\
\hline
\end{tabular}

PSQI: Pittsburgh Sleep Quality Index, Values are mean \pm M.S.E. M2 (age $>17-18$ years), M3 (age $>18-19$ years) and M4 (age $>19-20$ years) groups were compared with M1 (age 16-17 years) group. F2 (age $>17-18$ years), F3 (age $>18-19$ years), and F4 (age $>19-20$ years), groups were compared with F1 (age 16-17 years), group. Female (F) groups were compared with respective (similar age) male (M) groups.

\subsection{Sleep Duration (PSQI Category 3)}

Female groups (F1-F4) of nursing students showed significantly different $(P<0.005)$ mean score of sleep duration (PSQI category 3) in comparison to similar respective age male (M1-M4) groups. While both male and female groups remained insignificantly different from each other in the same sex (Table 3). 
Table 3. Comparison of sleep duration, aggregated score from the PSQI category 3 among male verses female nursing students

\begin{tabular}{lllll}
\hline Groups & Sex & Aggregated score & p-value & CI 95\% \\
\hline M1 (13) & Male & $2.08 \pm 0.212$ & & \\
F1 (20) & Female & $2.64 \pm 0.160 *$ & 0.0404 & $\begin{array}{l}-1.09393 \text { to } \\
\text { M2 (14) }\end{array}$ \\
Male & $2.13 \pm 0.120$ & & \\
F2 (20) & Female & $2.62 \pm 0.212^{*}$ & 0.0340 & -0.94064 to \\
M3 (14) & Male & $2.21 \pm 0.165$ & & -0.03936 \\
F3 (19) & Female & $2.74 \pm 0.157 *$ & 0.0290 & -1.00203 to \\
M4 (15) & Male & $2.09 \pm 0.289$ & & -0.05797 \\
F4 (18) & Female & $2.62 \pm 0.239 *$ & 0.0280 & -0.99902 to \\
\hline
\end{tabular}

PSQI: Pittsburgh Sleep Quality Index, Values are mean \pm M.S.E. M2 (age $>17-18$ years), M3 (age $>18-19$ years) and M4 (age $>19-20$ years) groups were compared with M1 (age 16-17 years) group. F2 (age $>17-18$ years), F3 (age $>18-19$ years), and F4 (age $>19-20$ years), groups were compared with F1 (age 16-17 years), group. Female (F) groups were compared with respective (similar age) male (M) groups.

\subsection{Sleep Efficiency (PSQI Category 4)}

Table 4 showed the mean score values of sleep efficiency of nursing students. Comparison of female groups to respective similar age male groups showed significantly different $(P<0.005)$ score. Score values obtained from M2, M3 and M4 found insignificantly different in comparison to the values of M1. Similarly sleep efficiency score values of female F2, F3 and F4 groups remained insignificantly different from values of F1 group (Table 4).

Table 4. Comparison of sleep efficiency, aggregated score from the PSQI category 4 among male verses female nursing students

\begin{tabular}{lllll}
\hline Groups & Sex & Aggregated score & p-value & CI 95\% \\
\hline M1 (13) & Male & $1.35 \pm 0.158$ & & \\
F1 (20) & Female & $1.84 \pm 0.124^{*}$ & 0.0199 & $\begin{array}{l}-0.89722 \text { to } \\
\text { M2 (14) }\end{array}$ \\
Male & $1.43 \pm 0.167$ & & -0.08278 \\
F2 (20) & Female & $1.88 \pm 0.116^{*}$ & 0.0289 & -0.85053 to \\
M3 (14) & Male & $1.51 \pm 0.115$ & & -0.04947 \\
F3 (19) & Female & $1.90 \pm 0.118^{*}$ & 0.0282 & -0.73545 to \\
M4 (15) & Male & $1.42 \pm 0.129$ & & -0.04455 \\
F4 (18) & Female & $1.92 \pm 0.140^{*}$ & 0.0147 & -0.89455 to \\
\hline
\end{tabular}

PSQI: Pittsburgh Sleep Quality Index, Values are mean \pm M.S.E.

M2 (age $>17-18$ years), M3 (age $>18-19$ years) and M4 (age $>19-20$ years) groups were compared with M1 (age 16-17 years) group. F2 (age $>17-18$ years), F3 (age $>18-19$ years), and F4 (age $>19-20$ years), groups were compared with F1 (age 16-17 years), group. Female (F) groups were compared with respective (similar age) male (M) groups.

\subsection{Sleep Disturbances (PSQI Category 5)}

Female groups (F1-F4) of nursing students showed insignificantly different $(P>0.005)$ mean score of sleep disturbances (PSQI category 5 ) in comparison to respective similar age male (M1-M4) groups. Further same sex groups did not differ from each other (Table 5).
Table 5. Comparison of sleep disturbance, aggregated score from the PSQI category 5 among male verses female nursing students

\begin{tabular}{lllll}
\hline Groups & Sex & Aggregated score & p-value & CI 95\% \\
\hline M1 (13) & Male & $1.98 \pm 0.270$ & & \\
F1 (20) & Female & $2.12 \pm 0.232$ & 0.7008 & $\begin{array}{l}-0.87621 \text { to } \\
0.59621\end{array}$ \\
M2 (14) & Male & $2.03 \pm 0.241$ & & -0.69926 to \\
F2 (20) & Female & $2.12 \pm 0.185$ & 0.7654 & 0.51926 \\
M3 (14) & Male & $2.10 \pm 0.264$ & & -0.84521 to \\
F3 (19) & Female & $2.15 \pm 0.272$ & 0.8988 & 0.74521 \\
M4 (15) & Male & $2.04 \pm 0.227$ & & -0.83544 to \\
F4 (18) & Female & $2.18 \pm 0.247$ & 0.6842 & 0.55544 \\
\hline
\end{tabular}

PSQI: Pittsburgh Sleep Quality Index, Values are mean \pm M.S.E.

M2 (age $>17-18$ years), M3 (age $>18-19$ years) and M4 (age $>19-20$ years) groups were compared with M1 (age 16-17 years) group. F2 (age $>17-18$ years), F3 (age $>18-19$ years), and F4 (age $>19-20$ years), groups were compared with F1 (age 16-17 years), group. Female (F) groups were compared with respective (similar age) male (M) groups.

\subsection{Use of Hypnotics/Anti-Depressants (PSQI Category 6)}

The use of hypnotics/anti-depressants mean score from the PSQI category 6 in nursing students did not differ from female groups verses respective similar age male groups. Groups of same sex did not differ from each other as well for the PSQI category 6 (Table 6).

Table 6. Comparison of use of hypnotics/anti-depressants, aggregated score from the PSQI category 6 among male verses female nursing students

\begin{tabular}{llllll}
\hline Groups & Sex & Aggregated score & p-value & CI 95\% & \\
\hline M1 (13) & Male & $1.07 \pm 0.125$ & & & \\
& & & & -0.66918 & to \\
F1 (20) & Female & $1.32 \pm 0.144$ & 0.2330 & 0.16918 & \\
M2 (14) & Male & $1.10 \pm 0.132$ & & -0.58464 & to \\
F2 (20) & Female & $1.30 \pm 0.128$ & 0.2975 & 0.18464 & \\
M3 (14) & Male & $1.05 \pm 0.122$ & & -0.69853 & to \\
& & & & 0.15853 & \\
F3 (19) & Female & $1.32 \pm 0.156$ & 0.2083 & & \\
& & & & & \\
M4 (15) & Male & $1.21 \pm 0.145$ & & 0.57820 & to \\
& & & & & \\
F4 (18) & Female & $1.34 \pm 0.160$ & 0.5584 & 0.31820 & \\
\hline
\end{tabular}

PSQI: Pittsburgh Sleep Quality Index, Values are mean \pm M.S.E.

M2 (age $>17-18$ years), M3 (age $>18-19$ years) and M4 (age $>19-20$ years) groups were compared with M1 (age 16-17 years) group. F2 (age $>17-18$ years), F3 (age $>18-19$ years), and F4 (age $>19-20$ years), groups were compared with F1 (age 16-17 years), group. Female (F) groups were compared with respective (similar age) male (M) groups.

\subsection{Daytime Dysfunction (PSQI Category 7)}

All male (M1-M4) and female (F1-F4) groups of nursing students showed mean score of daytime dysfunction insignificantly different $(P>0.005)$ from each other (Table 7). 
Table 7. Comparison of daytime dysfunctions, aggregated score from the PSQI category 7 among male verses female nursing students

\begin{tabular}{lllll}
\hline Groups & Sex & Aggregated score & p-value & CI 95\% \\
\hline M1 (13) & Male & $1.80 \pm 0.141$ & & -0.69111 to \\
F1 (20) & Female & $2.05 \pm 0.148$ & 0.2565 & 0.19111 \\
M2 (14) & Male & $1.73 \pm 0.313$ & & -1.34941 to \\
F2 (20) & Female & $2.12 \pm 0.327$ & 0.4138 & 0.56941 \\
M3 (14) & Male & $1.92 \pm 0.247$ & & -1.08380 to \\
F3 (19) & Female & $2.14 \pm 0.314$ & 0.6071 & 0.64380 \\
M4 (15) & Male & $1.96 \pm 0.283$ & & -1.10588 to \\
F4 (18) & Female & $2.22 \pm 0.296$ & 0.5353 & 0.58588 \\
\hline
\end{tabular}

PSQI: Pittsburgh Sleep Quality Index, Values are mean \pm M.S.E.

M2 (age $>17-18$ years), M3 (age $>18-19$ years) and M4 (age $>19-20$ years) groups were compared with M1 (age 16-17 years) group. F2 (age $>17-18$ years), F3 (age $>18-19$ years), and F4 (age $>19-20$ years), groups were compared with F1 (age 16-17 years), group. Female (F) groups were compared with respective (similar age) male (M) groups.

\section{Discussion}

In the present study, nursing students enrolled in the School of nursing and midwifery of MBU in Tehran, Iran, reported a score point approximately 2.09 which means a fairly poor subjective sleep quality (overall total score $=14.6125 \pm 1.38$ ). The statistical analysis of the score obtained in each category from the PSQI also shows that nursing students present a fairly bad subjective sleep quality. Statistical analysis shows a significantly higher $(\mathrm{P}=0.0305$, CI $95 \%=-6.685494$ to -0.334506$)$ total mean score of female student $(16.3675 \pm 1.15, \mathrm{nf}=77)$ versus male students $(12.8575 \pm 1.02, \mathrm{~nm}=56$, Fig. 1 and 2$)$. Male students present score point 1.85 and female students 2.34 . Higher score among female students indicate a poorer level of sleep quality. Students generally exhibit poor sleep quality [21], which has been associated to insomnia and other sleep disturbances, to the high course load [7] and to demographic and hygiene behaviors [21]. In the present study, PSQI category 1 presents a score, 2.65 points (approximately, Fig. 3). Female groups' comparison to the similar age male groups shows the significantly higher mean scores (Table 1), suggesting poorer quality of sleep in female than male students. This finding is in consistent with earlier reports [22-23]. Where, the reduced sleep duration in students (i.e., a total sleep duration of 5 to 6 hours on average) was found to be related to the class schedules [24] and the high course load [7]. Another study [25] revealed a gender differences in circadian typology and in biological rhythms. Data also indicates a gender difference in the subjective sleep duration, PSQI category 2 which presents a score, nearly 2.81 points (Fig. 3, Table 2). Moreover, sleep deprivation in students impairs the academic and professional performances [26]. In this study, nursing students from the School of nursing and midwifery of MBU in Tehran, Iran, sleep 5 to 6 hours on average, which yields a score near from 2.72 points in the PSQI scale (Fig. 3). Considering that the maximum score (3 points) of the PSQI scale means the worst sleep quality (6), and that students show a higher sleep deprivation [7-24]. While a significant difference among female versus male nursing students indicates greater sleep deprivation in female than male participants (Table 3 and 4). Daytime dysfunctions, mainly excessive daytime sleepiness, have been described in the students [7-27]. Moreover, excessive daytime sleepiness in the students has been correlated with short sleep duration [13], poor academic performance [12], minor psychiatric disorders [10], sleep disorders [16], depressive symptoms [17] and poor sleep quality [7]. According to our findings, daytime dysfunctions also affect the nursing students from School of nursing and midwifery of MBU in Tehran (Fig.3). Although there is no significant difference observe on gender basis (Table 5-7). However, PSQI categories 5, 6 and 7 score point (2.39, 1.39 and 2.28 respectively) indicates a bad quality of sleep. As daytime dysfunctions impair the student daytime activities and academic performance [8-29], applying an educative approach that attenuates the occurrence of these disorders, may improve the student learning abilities.

\section{Conclusion}

In brief, the present study shows that nursing students from School of nursing and midwifery of MBU in Tehran, presents a fairly poor subjective sleep quality and a slight sleep deprivation and reports a fairly high incidence of daytime dysfunctions. When compare to female versus male a more poor sleep quality correlates with poor health level of female students than the male students, findings allow to suggest that improvement in sleep quality in nursing institutes may contribute to avoid further damages in students health and thus, their academic performance. Sleep is a homeostatic process with clear effects on wellbeing, cognition, attention and behavior. The implementation of a program to aid the students, in order to promote sleep hygiene is suggested. While this is the personal opinion of the present author rather than a direct conclusion of the study, such a measure could result in better academic results and better professional performance.

\section{Appendix A: Pittsburgh Sleep Quality Index (PSQI)}

\section{Instructions}

The following questions related to your usual sleep habits during the past month only. Yours answers should indicate the most accurate reply for the majority of days and nights in the past month. Please answer all questions.

1. During the past month, when have you usually gone to bed at night?

USUAL BED TIME

2. During the past month, how long (in minutes) has it 
usually take you to fall asleep each night?

NUMBER OF MINUTES

3. During the past month, when have you usually gotten up in the morning?

\section{USUAL GETTING UP TIME}

4. During the past month, how many hours of actual sleep did you get at night? (This may be different than the number of hours you spend in bed).

HOURS OF SLEEP PER NIGHT

For each of the remaining questions, check the one best response. Please answer all questions.

5. During the past month, how often have you had trouble sleeping because you...

(a) Cannot get to sleep within 30 minutes

Not during the past month

Once or twice a week Less than once a week week Three or more times a

(b) Wake up in the middle of the night or early morning

Not during the past month Once or twice a week Less than once a week week Three or more times a

(c) Have to get up to use the bathroom

Not during the past month Less than once a week week Once or twice a week Three or more times a

(d) Cannot breathe comfortably

Not during the past month Once or twice a week Less than once a week week Three or more times a

(e) Cough or snore loudly

Not during the past month week

Once or twice a week

Less than once a week

(f) Feel too cold

Not during the past month Once or twice a week week Three or more times a

(g) Feel too hot

Not during the past month Once or twice a week week Less than once a week Three or more times a

(h) Had bad dreams

Not during the past month Once or twice a week Less than once a week Three or more times a week

(i) Have pain Not during the past month once a week Once or twice a week Less than once a week Three or more times a more times a week

(j) Other reason(s), please describe

6. During the past month, how would you rate your sleep quality overall?

Very good Fairly good Fairly bad Less than bad

7. During the past month, how often have you taken medicine (prescribed or "over the counter") to help you sleep?

Not during the past month Once or twice a week Less than once a week week Three or more times a

8. During the past month, how often you had trouble staying awake while driving, eating meals, or engaging in social activity?

Not during the past month Once or twice a week Less than once a week week Three or more times a

9. During the past month, how much of a problem has it been for you to keep up enough enthusiasm to get things done?

Not problem at all_only a very slight problem Somewhat of a $\overline{\text { problem }}$ A very big problem

10. Do you have a bed partner or roommate?

No bed partner or roommate___ Partner/roommate in other room Partner in same room, but not same bed Partner in same bed

If you have a roommate or bed partner, ask him/her how often in the past month you have had... (a) Loud snoring Not during the past month Once or twice a week Less than once a week week Three or more times a

(b) Long pauses between breaths while asleep

Not during the past month Once or twice a week Less than once a week week Three or more times a

(c) Legs twitching or jerking while you sleep

Not during the past month Less than once a week week Once or twice a week Three or more times a

(d)Episodes of disorientation or confusion during sleep

Not during the past month Once or twice a week Less than once a week week Three or more times a

(e) Other restlessness while you sleep, please describe Not during the past month week Once or twice a week Less than once a week

\section{Acknowledgments}

Sincere thanks go to: The author would like to express his sincere thanks to Student Affairs Center of Martyr Beheshti University of Medical Sciences and Health Services, Mehr, Jam and Kasra Hospitals in Tehran, Iran.

\section{References}

[1] NIH. National center on sleep disorders research plan. 2011. NIH Publication $\quad$ No. 11-7820. [http://www.nhlbi.nih.gov/health/prof/sleep/201101011Natio nalSleepDisordersResearchPlanDHH SPublication117820.pdf] 
[2] Chokroverty S. Overview of sleep and sleep disorders. Indian J Med Res. 2010;131:126-40.

[3] Reeth VO, Weibel L, Spiegel K, Leproult R, Dugovic C, Maccari S. Physiology of sleep (eview)- Interactions between stress and sleep: from basic research to clinical situations. Sleep Medicine Reviews. 2000;4(2):201-19.

[4] Desseilles M, Dang-Vu TT, Sterpenich V, Schwartz S. Cognitive and emotional processes during dreaming: a neuroimaging view. Conscious Cogn. 2011;20(4):998-08.

[5] Castro ECP, Figueiredo FF, Lima GAF, Amorim HF, Bastos LMH, de-Moraes PHA, de-Abreu- Balan TJ, Junior MF, Restini CBA. Evaluation of the sleep quality in medical students from Ribeirão Preto, SP / Brazil. Scholarly J Med. 2012;2(4):40-50.

[6] Diekelmann S, Born J. The memory functions of sleep. Nat Rev Neurosci. 2010;11:114-26.

[7] Cardoso HC, Bueno FCC, da-Mata JC, Alves APR, Jochims I, Vaz-Filho IHR, Hanna MM. Assessing quality of sleep in medical students. Rev Bras Ed Med. 2009;33:349-55.

[8] Furlani R, Ceolim MF. Padrões de sono de estudantes ingressantes na Graduação em Enfermagem. Rev Bras Enferm. 2005;58:320-4.

[9] Onyper SV, Thacher PV, Gilbert JW, Gradess SG. Class start times, sleep, and academic performance in college: a path analysis. Chronobiol Int.2012;29:318-35.

[10] Coelho AT, Lorenzini LM, Suda EY, Rossini S, Reimao R. Sleep quality, depression and anxiety in college students of last semesters in health area courses. Neurobiologia. 2010;73:35-9.

[11] Nojomi MN, Bandi MFG, Kaffashi S. Sleep pattern in medical students and residents. Arch Iran Med. 2009;12:542-9.

[12] Rodrigues RN, Viegas CA, Abreu E, Silva AA, Tavares P. Daytime sleepiness and academic performance in medical students. Arq Neuropsiquiatr. 2002;60:6-11.

[13] Bahammam AS, Al-Khairy OK, Al-Taweel AA. Sleep habits and patterns among medical students. Neurosciences (Riyadh). 2005;10:159-62.

[14] Aloe F, Pedroso A, Tavares SM. Epworth sleepiness scale outcome in 616 Brazilian medical students. Arq Neuropsiquiatr. 1997;55:220-6.

[15] Zailinawati AH, Teng CL, Chung YC, Teow TL, Lee PN, Jaqmohni KS. Daytime sleepiness and sleep quality among Malaysian medical students. Med J Malaysia. 2009;64:10810.

[16] Veldi M, Aluoja A, Vasar V. Sleep quality and more common sleep-related problems in medical students. Sleep Med. 2005;6:269-75.

[17] Eller T, Aluoja A, Vasar V, Veldi M. Symptoms of anxiety and depression in Estonian medical students with sleep problems. Depress Anxiety. 2006;23:250-6.

[18] Subramaniam RM. Problem-based learning: concept, theories, effectiveness and application to radiology teaching. Australas Radiol. 2006;50:339-41.

[19] Mesquita G, Reimão R. Nightly use of computer by adolescents. Its effect on quality of sleep. Arq Neropsiquiatr. 2007;65:428-32.

[20] Zaman R. Prevalence of Hepatitis B and Hepatitis C viruses in human urban population of Bahawalpur District, Pak. J Med Sci. 2006;6(3):429-35.

[21] Adeosun SO, Asa SO, Babalola OO, Akanmu MA. Effects of night-reading on daytime sleepiness, sleep quality and academic performance of undergraduate nursing students in Nigeria. Sleep and Biological Rhythms. 2008;6(2):91-4.

[22] Middelkoop HAM, den-Doel DAS, Neven AK, Kamphuisen HAC, Springer P. Subjective Sleep Characteristics of 1,485 Males and Females Aged 50-93: Effects of Sex and Age, and Factors Related to Self-Evaluated Quality of Sleep. J Gerontol A Biol Sci Med Sci. 1996; 51A(3):M108- M115.

[23] Medeiros ALD, Mendes DBF, Lima PF, Araujo JF. The Relationships between Sleep-Wake Cycle and Academic Performance in Medical Students. Biological Rhythm Research. 2001;32(2):263- 70.

[24] Medeiros ALD, Araujo JF. Sleep-wake pattern of medical students: early versus late class starting time. Braz J Med Biol Res. 2002;35(11):1373-7.

[25] Randler C. Gender differences in morningness-eveningness assessed by self-report questionnaires: A meta-analysis. Personality and Individual Differences. 2007;43(7):1667-75.

[26] Danda GJN, Feereira GR, Azenha M, Souza KFR, Bastos O. Padrão do ciclo sono-vigília e sonolência excessiva diurna em estudantes de medicina. J Brás Psiquiatr. 2005;54:102-6.

[27] Ioachimescu OC, El-Solh AA. Pharmacotherapy of insomnia. Expert Opin Pharmacother . 2012;13(9):1243-60.

[28] dos Passos TES, Minotelli RG, Koeke RS, Proto VJ, Spada SA Jr. Quality of sleep and daytime sleepiness among university students. J Sleep Disor: Treat Care. 2013;2:2.

\section{Biography}

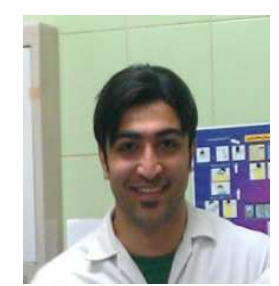

Morteza Alibakhshi-kenari was born in February 1989 at Iran, Mazandaran, Babolsar. He received the B.S. degrees from the Sari University of Medical Sciences and Health Services at Iran, in February 2011, and he is now a Ph.D student at the martyr Beheshti University of Medical Sciences and Health Services, Tehran, Iran. He is currently working in the field of Evidence Base Nursing. 